\title{
High Quality Heart and Lung Auscultation System for Diagnostic Use on Remote Patients in Real Time
}

\author{
Roberto Marani, Gennaro Gelao and Anna Gina Perri*
}

\author{
Politecnico di Bari, Dipartimento di Elettrotecnica ed Elettronica, Laboratorio di Dispositivi Elettronici, Via E. \\ Orabona 4, 70125, Bari, Italy
}

\begin{abstract}
We propose a medical electronic-computerized platform for diagnostic use, which allows doctors to carry out a complete cardio-respiratory control on remote patients in real time. In the context of telemedicine the proposed system can be considered as a really innovative product in which all the most advanced technologies of biomedical engineering converge to guarantee an efficient and reliable home assistance that allows the patient a highly better quality of life in terms of prophylaxis, treatment and reduction of discomfort connected to periodic patient controls and/or hospitalization. Moreover the system has been equipped to be employed also to real-time rescue in case of emergency without the necessity for data to be constantly monitored by a medical centre. In fact, when an emergency sign is detected through the real-time diagnosing system, it sends a warning message to people able to arrange for his/her rescue. A Global Positioning System (GPS) also provides the patient coordinates. The proposed system, in its version for diagnostic use, has been verified by the heart specialists of the Institute of Cardiology in the General Hospital (Polyclinic) of the University of Bari, Italy.
\end{abstract}

Keywords: Electronic medical devices, electrocardiogram and respiratory monitoring, health care management systems.

\section{INTRODUCTION}

The most recent developments of electronics, informatics and telecommunications let imagine applications in the biomedical engineering field to improve the healthcare quality [1]. In particular a number of systems has been developed in the telemedicine and home care sectors which could guarantee an efficient and reliable home assistance allowing a highly better quality of life in terms of prophylaxis, treatment and reduction of discomfort connected to periodic out-patient controls and/or hospitalization for the patients afflicted by pathologies (such as cardiac decompensation or obstructive chronic bronchopathy), and allowing considerable savings on sanitary expenses.

Although there are already available instruments for the remote monitoring of the ElectroCardioGram (ECG) [2], the contemporary Cardiac and Pulmonary tele-Auscultation (ACP) is not carried out yet for the lack of adequate instruments and clinical validation of the methods.

However the known tele-electrocardiographs are able to transfer the electrocardiograms only after the acquisition, not in real time and are mostly and strongly orientated towards the sanitary emergencies. In fact they are typically installed on ambulances and need a skilled staff for the utilization [34].

On the other hand it is essential to observe the electrocardiogram is not the only source of information useful to evaluate the patient health.

\footnotetext{
*Address correspondence to this author at the Politecnico di Bari, Dipartimento di Elettrotecnica ed Elettronica, Laboratorio di Dispositivi Elettronici, Via E. Orabona 4, 70125, Bari, Italy; Tel: +39-805963314/5963427; Fax: +39-80-5963410; E-mail: perri@ poliba.it
}

It is obvious, therefore, that there is a rather limited offer of the current market with regard to the requirements which a health service should meet, if it is in the lead with regard to the effective potentialities offered by the present technology.

Particularly we recognize deficiency or total absence of reliable and valid telemedicine platforms which allow the follow up of patients and the acquisition of the main vital parameters, such as electrocardiograms, spirometry, oximetry, cardiac tones, lung sounds, with a doctor in a different place regard to the patient.

In this paper we propose a medical electroniccomputerized platform for diagnostic use, which allows the doctor to carry out a complete cardio-respiratory control on remote patients in real time. The system has been designed to be employed also to real-time rescue in case of emergency without the necessity for data to be constantly monitored by a medical centre, leaving patients free to move. For this purpose the system has been equipped with highly developed firmware which enables automated functioning and complex decision-making. In fact, when an emergency sign is detected through the real-time diagnosing system, the system sends a warning message to persons able to arrange for his/her rescue, providing also the patient's coordinates. All this occurs automatically without any intervention by the user. The system might be useful also to sportsmen.

Finally the system, in its version for diagnostic use, has been verified by the heart specialists of the Institute of Cardiology in the General Hospital (Polyclinic) of the University of Bari, Italy.

\section{MAIN CHARACTERISTICS}

The designed system is a medical electronic informational platform for diagnostic use, which permits the 
doctor to carry out a complete cardio-respiratory control on remote patients in real time.

In fact, as if the doctor is present personally near the patient, the system allows him to receive the following data in real time:

1. auscultation of cardiac tones and broncho-pulmonary sounds

2. electrocardiogram

3. arterial blood pressure

4. oximetry

5. respiration frequency

6. phonocardiography

7. spirometry

8. image and audio of the patient with high quality.

The system consists of two parts: a patient station and a doctor position, both compact and light easily transportable, both are composed of committed laptop, hardware and software.

The patient unit is equipped with miniaturized diagnostic instruments and is suitable also for paediatrics use. Many patient stations can correspond to one doctor position.

The system is modular and allows to select and to install some of the suitable diagnostic instruments, even though it is prearranged for the plug and play installation of the others (for example only the electrocardiograph can be installed and then also the phonendoscope, etc.).

The electrocardiogram could record up to 12 derivations and the software is able to interpret the data and to automatically carry out the reading and the diagnosis of the trace which should be confirmed by the doctor. It is possible to carry out monitoring without time limits and always in real time. This makes possible the capture of uneven heartbeats or also intermittent ones of other nature. The acquire trace is registered and filed.

The tele-phonendoscope is of electronic kind and obtains biological sounds in the $20 \mathrm{~Hz}-1 \mathrm{kHz}$ band and can be used in three modes in order to improve the cardiac and pulmonary auscultation: membrane, bell and extensive modality. Moreover, it allows the $75 \%$ suppression of the external noise.

It is equipped with software for the real time spectrum analysis and it starts automatically at the beginning of the auscultation procedure. The positioning of the phonendoscope is led by a remote doctor thanks to the full time audio/video communication and the biological sounds can be simultaneously heard either by the patient (or by an operator helping the patient in the examination) or by the doctor in remote.

The biological sounds are also registered during the acquisition with significant advantages for diagnosis accuracy and for possibility of carrying out diagnostic comparisons with previous records.

The tele-spirometer allows to carry out the FVC, VC, MVV tests and to determine the respiratory frequency and it is autodiagnostic.
The finger (optic) tele-saturimeter allows to carry out the monitoring (check without time limit) of the $\mathrm{SpO}_{2}$ value as it is equipped with plug-in which permits the tracing of the saturation values curve that will be presented in real time to the doctor.

The filing of the data concerning the carried out examination occurs in a dynamic database both on the patient position and on the doctor position; the data will be filed by ordering them for each patient.

Thus to each patient a clinical record will be associated containing all his data. This kind of filing is very useful to carry out diagnostic comparisons on the evolution of a disease or on the outcome of a therapy, and it eases him of the burden of having the record documentation regarding him personally. In the patient data base there is also a filed schedule containing the personal details of the patient, the case history in addition to various notes, values of blood tests, the outcome of other diagnostic tests, treatments undertaken during the time, therapy in course, etc.

This system also makes possible to transmit echograms, $\mathrm{X}$-rays radiograms and other tests in digital form to the doctor and also their filing in the patient database.

The doctor can also prescribe other subsequent clinical tests advised and/or treatments to undertake.

The system does not present connectivity limits of any kind find and requires a $320 \mathrm{~Kb} / \mathrm{s}$ minimum band or a UMTS Mobile telephone.

The system has an user friendly software interface very easy to be used, because it implements the one touch philosophy, and requires extremely reduced operating costs.

The patient can ask for a medical examination and the doctor can accept or refuse to examine him if busy. As a result of the doctor availability, the medical examination can start and the doctor can ask for the necessary tests through a simple "click".

This system has been planned/designed in the observance of the current regulations for medical devices, informatic safety and privacy.

The system, therefore, is marked by three distinct and basic fundamental characteristics:

1. the real time data transmission by assuring the remote doctor the simultaneous control of the data during their acquisition;

2. the possibility to carry out a complete telematic medical examination, including the tele-auscultation, all the operations the doctor performs when he examines the patient directly at home or at the surgery and even more, since the system is equipped with typically diagnostic instruments not available at the family doctor's but at hospital units;

3. the possibility to establish a continuous audio/video communication during the examination, in order that the same doctor can interact with the patient, verifying the correct positioning of the sensors and having also a very high quality image of the patient, which can be useful for diagnostic aims. 
Among the most evident and important applications we can indicate the following ones:

1. home tele-assistance of cardiac patients in decompensation or of chronic patients with pathologies attributed to the cardio-circulatory or respiratory apparatus;

2. mass prophylaxis with complete cardio-respiratory control, frequently and at low cost;

3. tele-consultation;

4. follow-up of patients discharged early (precociously) and in need of tele-protection;

5. closed-circuit monitoring of the health of patients waiting for hospitalization.

The reduction of hospitalization time, using home teleprotection, and the avoided hospitalization of patients in decompensation monitored at home imply large economic saving. The shorter patient presence in hospitals reduce the waiting lists in a remarkable way.

\section{FURTHER APPLICATION OF THE SYSTEM FOR CONTINUOUS HEALTH MONITORING}

Today there is a growing need for inexpensive and reliable health monitoring devices [5], able to record data, to analyze them in real time and, if possible, to transmit them to a receiving unit by exploiting wireless technology, but the market still does not seem to offer any reliable GPRS or Bluetooth-based, effective and low-cost health-monitoring telemetric systems.

Although telemetric systems are already used in hospitals, they do not seem to fully exploit all the potential of modern technology and seem to suffer from some important limitations. In fact, many devices are specifically intended for emergencies [6] and can transmit ECG results, as well as those from the monitoring of some other parameters. Such systems are inadequate for continuous health monitoring, not easy to use and have to be managed only by qualified operators, which makes them unsuited for personal use and domestic applications.

Moreover one of the limitations of existing devices lies in the fact that they are not wearable and allow only to monitor ECG, saturation and some other parameters separately, otherwise extraordinary bandwidth would be needed in order to transfer all data, especially via GSM (at the limited speed of $9200 \mathrm{kbit} / \mathrm{s}$ ). GPRS and UMTS technologies result from the development of GSM transmission of packet data.

The combination of the latest suitable telecommunication solutions (GPRS and Bluetooth) with new algorithms and solutions for automatic real-time diagnosis, costeffectiveness (both in terms of purchase expenses and data transmission/analysis) and simplicity of use (the patient will be able to wear it) can give the designed system useful for remote health monitoring, allowing real-time rescue operations in case of emergency without the necessity for data to be constantly monitored.

For this purpose the proposed system has been equipped with highly developed firmware which enables automated functioning and complex decision-making. It is indeed able to prevent lethal risks thanks to an automatic warning system. All this occurs automatically without any intervention by the user.

Each monitored patient is identified by a case sheet on a Personal Computer (PC) functioning as a server (online doctor). Data can also be downloaded by any other PC, palmtop or smartphone equipped with a browser. The system reliability rests on the use of a distributed server environment, which allows its functions not to depend on a single PC and gives more online doctors the chance to use them simultaneously.

The whole system consists of three hardware units and a management software properly developed. The units are:

- Elastic band: the sensors for the measurement of health parameters are embedded in an elastic band to be fastened round the patient's chest.

- $\quad$ Portable Unit (PU), which is wearable and wireless (GPRS/Bluetooth). This PU allows, by an Internet connection, the transmission, continuous or sampled or on demand, of the health parameters and allows the GPS satellite localization and the automatic alarm service, on board memory. Moreover PU has an USB port for data transfer and a rechargeable battery.

- Relocable Unit (RU): GPRS/Bluetooth Dongle (on $\mathrm{PC}$ server, i.e. online doctor).

- Management Software: GPS mapping, address and telephone number of nearest hospital, simultaneous monitoring of more than one patient, remote (computerized) medical visits and consultation service, creation and direct access to electronic case sheets (login and password)

Fig. (1) shows a picture of the PU. The very small dimensions are remarkable, even if it is only a prototype, realized at the Electronic Devices Laboratory of Polytechnic of Bari, and more reduction in dimensions is still possible.

The system, in particular the PU, collects data continuously. These are stored in an on-board flash memory and then analyzed real-time by an on-board automatic diagnosis software. Data can be sent to the local receiver, directly to the PC server (online doctor), or to an internet server, which allows anyone to download them once identified with his/her own login and password.

Data can be transmitted as follows:

1. real time continuously

2. at programmable intervals (for 30 seconds every hour, for example)

3. automatically, when a danger is identified by the alarm system

4. on demand, whenever required by the monitoring centre

5. offline (not real-time), downloading previously recorded (over 24 hours, for example) data to a PC.

In all cases patients do not need to do anything but simply switching on.

When an emergency sign is detected through the real time diagnosing system, the PU automatically sends a 


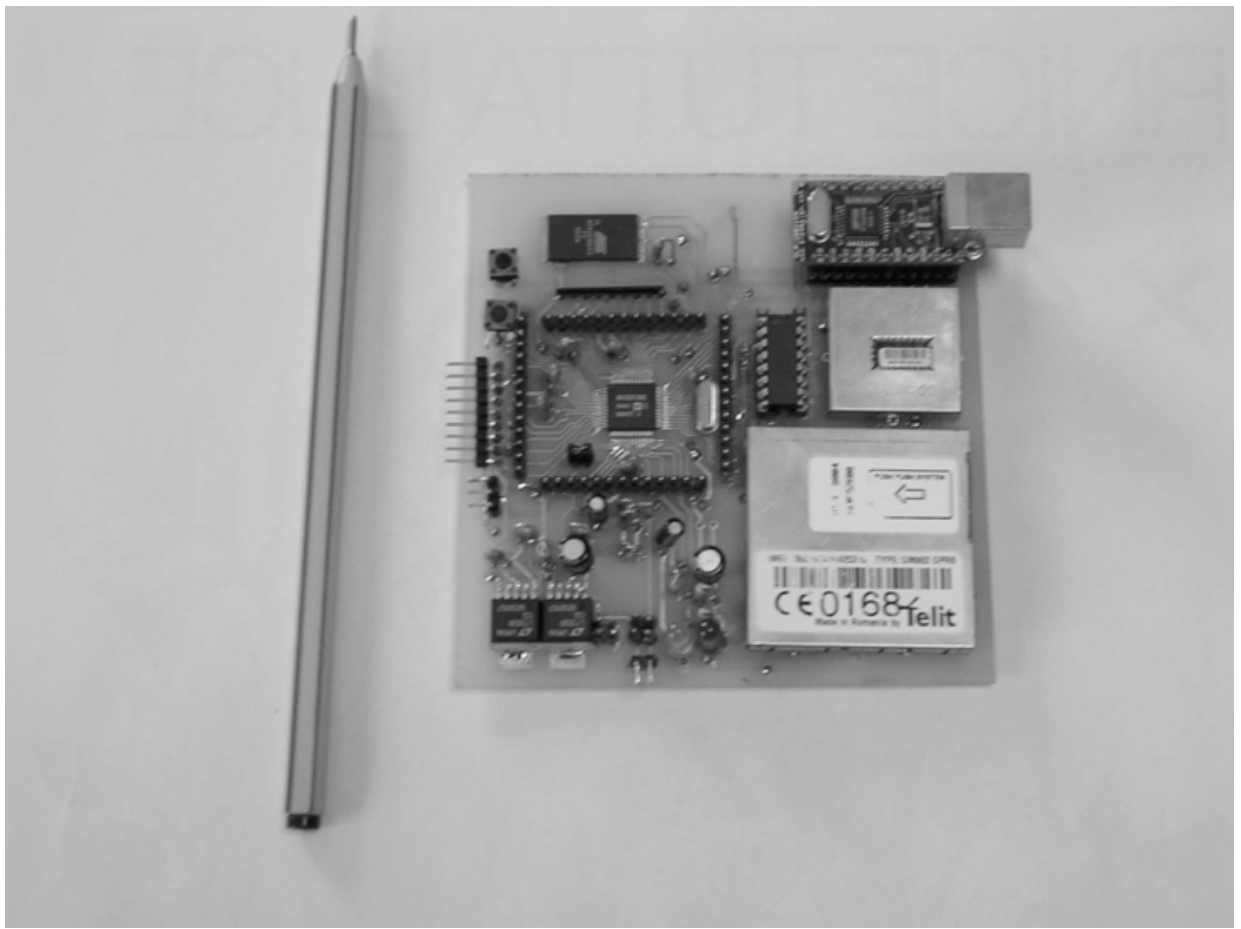

Fig. (1). A picture of the Portable Unit.

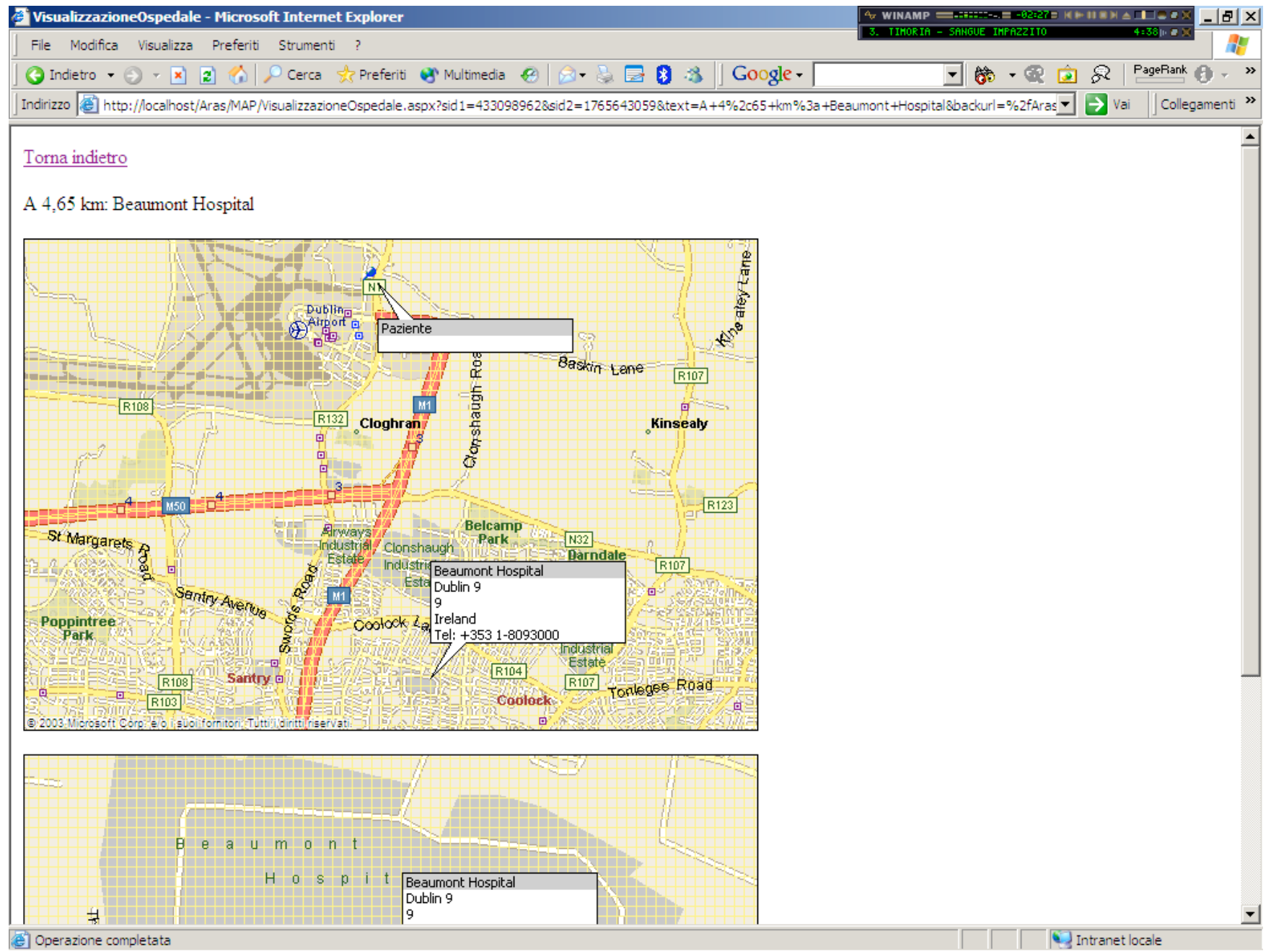

Fig. (2). GPS mapping with address and telephone number of nearest hospital. 
warning message, indicating also the diagnosis, to one person (or even more) who is able to verify the patient health status and arrange for his/her rescue. In order to make rescue operations as prompt as possible, the PU provides the patient coordinates using the GPS unit and the Management Software provides in real time a map indicating the position of the patient, as shown in Fig. (2).

Fig. (3) shows a picture of an electrocardiogram transmitted by Bluetooth and plotted on a Personal Computer by the proper developed management software.

\section{VALIDATION OF THE SYSTEM AND ANALYSIS OF THE RESULTS}

The proposed system, in its version for diagnostic use, has been used in a routine clinical context in order to evaluate the feasibility and accuracy of the ACP carried out via Internet compared with the traditional auscultation $(\mathrm{T})$.

At the Cardiac Decompensation Unit of Bari Polyclinic 21 patients (16 males, 5 females, in stable clinical conditions) have been examined. Each patient has been examined by two experienced heart specialists (Obs1 and Obs2) in two successive phases (I-ACP and T-ACP).

The I-ACP check-up has been carried out by the instrumentation which the system is equipped with. The audio signal of high quality obtained from the cardiopulmonary auscultation has been transmitted on internet through a standard ADSL connection from a patient position to a remote doctor position. The observer was able to listen and simultaneously record an audio file (WAV Format) of the ACP. A trained nurse placed the membrane on two auscultations spots under the visual guide of the doctor by means of a properly positioned webcam. The T-ACP checkup has been carried out with a traditional sound phonendoscope. The listened reports were recorded in a data file with preclassified reports. The obtained data have been analysed in terms of uniformity and examined with fisher's exact test $(\mathrm{p}<0,05)$ and the kappa-test (where applicable).

In Table 1 the data of the ACP carried out both via internet and in a traditional way have been shown for the observer 1 and for the observer 2 (intra-observer analysis).

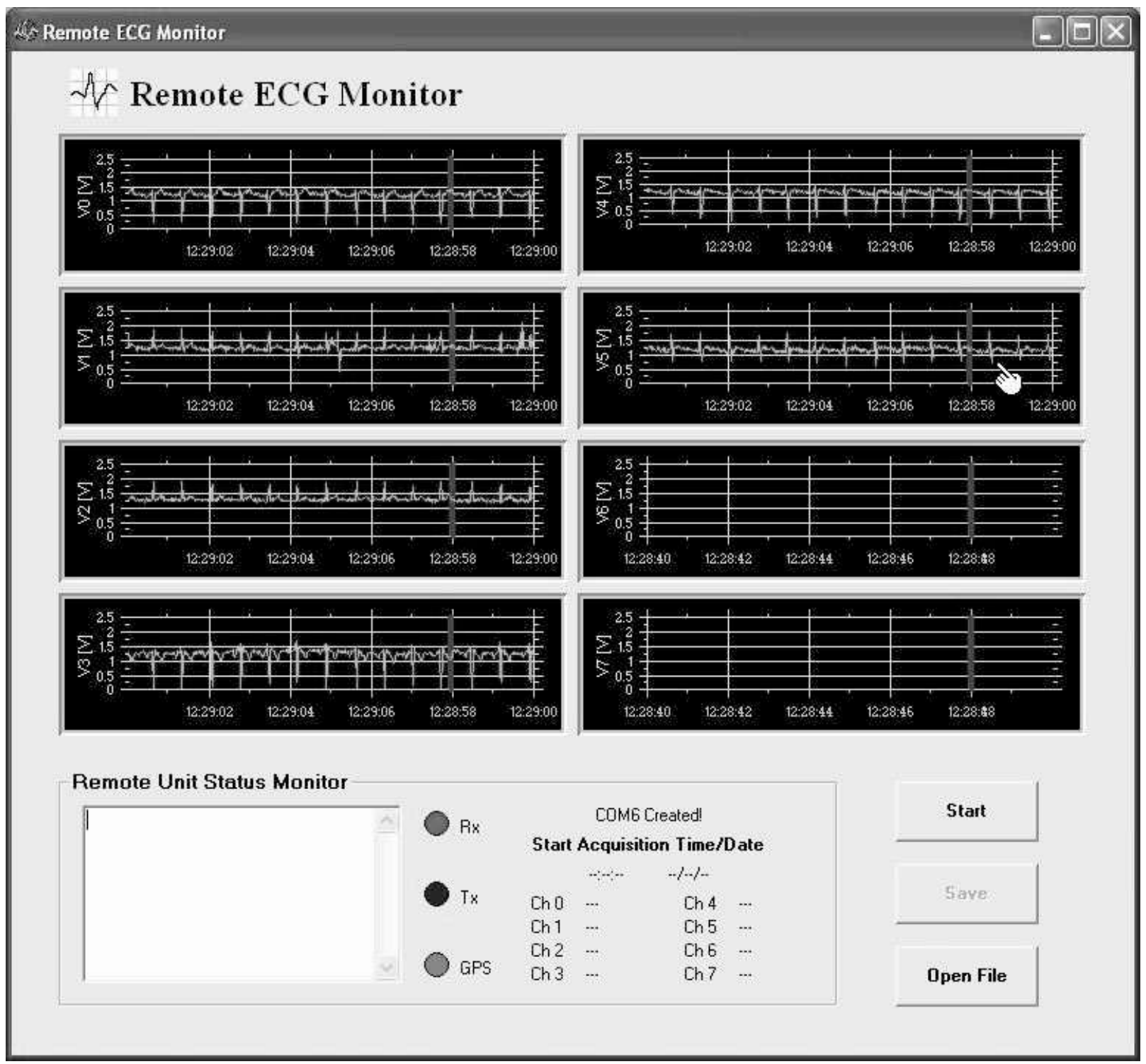

Fig. (3). Example of acquisition by Bluetooth of an electrocardiogram. 
Table 1. Data of the ACP Carried Out Both Via Internet and in a Traditional Way

\begin{tabular}{|c|c|c|c|c|}
\hline & \multicolumn{4}{|c|}{$\begin{array}{l}\text { Cardio pulmunary auscultation } \\
\text { Acoustic vs web } \\
\text { Intra-observer / inter-observer analysis }\end{array}$} \\
\hline & Obs 1 & Obs 2 & Web & Acoustic \\
\hline Total & $\begin{array}{l}217 / 231 \\
(93.9 \%)\end{array}$ & $\begin{array}{l}214 / 231 \\
(92.6 \%)\end{array}$ & $\begin{array}{l}224 / 231 \\
(97,0 \%)\end{array}$ & $\begin{array}{l}219 / 231 \\
(94.8 \%)\end{array}$ \\
\hline Cardiac & $\begin{array}{c}81 / 84 \\
(96.4 \%)\end{array}$ & $\begin{array}{c}79 / 84 \\
(94.0 \%)\end{array}$ & $\begin{array}{c}79 / 84 \\
(94,0 \%)\end{array}$ & $\begin{array}{c}83 / 84 \\
(98,8 \%)\end{array}$ \\
\hline Pulmonary & $\begin{array}{c}60 / 63 \\
(95.2 \%)\end{array}$ & $\begin{array}{c}59 / 63 \\
(93.7 \%)\end{array}$ & $\begin{array}{c}59 / 63 \\
(93.7 \%) \\
\end{array}$ & $\begin{array}{c}62 / 63 \\
(98.4 \%) \\
\end{array}$ \\
\hline $\begin{array}{l}\text { Cronology of } \\
\text { systolic } \\
\text { murmurs }\end{array}$ & $\begin{array}{l}19 / 21 \\
(90.5 \%) \\
k=0.82\end{array}$ & $\begin{array}{c}18 / 21 \\
(85.7 \%)\end{array}$ & $\begin{array}{c}17 / 21 \\
(81.0 \%)\end{array}$ & $\begin{array}{l}19 / 21 \\
(90.5 \%) \\
k=0.82\end{array}$ \\
\hline $\begin{array}{l}\text { Inspiratory } \\
\text { crepitations }\end{array}$ & $\begin{array}{c}20 / 21 \\
(95,2 \%) \\
k=0.64\end{array}$ & $\begin{array}{c}19 / 21 \\
(90.5 \%)\end{array}$ & $\begin{array}{c}20 / 21 \\
(95,2 \%)\end{array}$ & $\begin{array}{l}21 / 21 \\
(100 \%) \\
k=0.47\end{array}$ \\
\hline
\end{tabular}

Moreover, the data registered by the two observers according to the two different ways of check-up (intra-observer analysis) have been compared.

The intra-observer concordance of I-Vs IACP (number of concordant reports, (\%)) for the observer 1 and the observer 2 have been respectively:

- $\quad 217 / 231(93.9 \%)$ and $214 / 231(92.6 \%)(n s)$ for the total reports,

- $\quad 81 / 84(96.4 \%)$ and $79 / 84(94.0 \%)(n s)$ for the cardiac reports,

- 60/63 (95.2\%) and 59/63 (93.7\%) (ns) for the pulmonary reports.
The intra-observer concordance of observer 1 has been 19/21 $(90.5 \%$, kappa $=0.82)$ for the chronology of the systolic murmurs and 20/21 $(95.2 \%$, kappa $=0.64)$ for teleinspiratory crepitations.

The inter-observer concordances for I-Vs T-ACP have been respectively:

- $\quad 224 / 231(97.0 \%)$ versus 219/231 (94.8\%) (ns) for the total reports,

- $79 / 84(94.0 \%)$ versus $83 / 84(98.8 \%)(n s)$ for the cardiac reports,

- $\quad 59 / 63(93.7 \%)$ versus $62 / 63(98.4 \%)$ (ns) for the pulmonary reports.

The detailed analysis of each specific typology of report has been carried out.

The Figs. (4 and 5) show the histograms of the data taken with the intra-observer analysis and inter-observer one respectively.

The cardiac and pulmonary auscultation, evaluated in our series of cases through concordance analysis, has shown a high concordance of pulmonary and cardiac listener reports both for the traditional approach and for the telemedicine one via Internet.

The intra-observer and inter-observer concordances have not been significantly different for the two observers in the two operational contexts, showing that the cardiopulmonary auscultation with our system is an innovative diagnostic method able to improve the present procedures of telemonitoring.

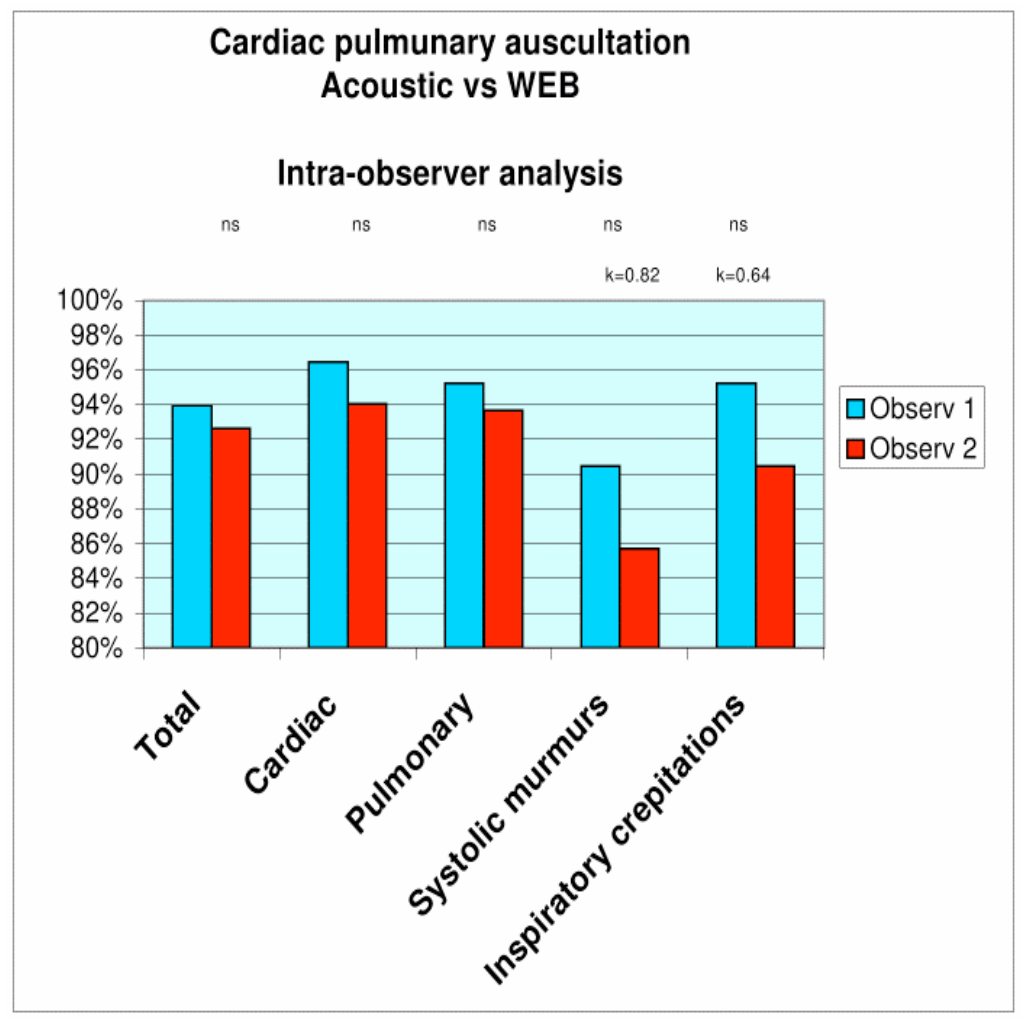

Fig. (4). Histogram of the data taken with the intra-observer analysis. 


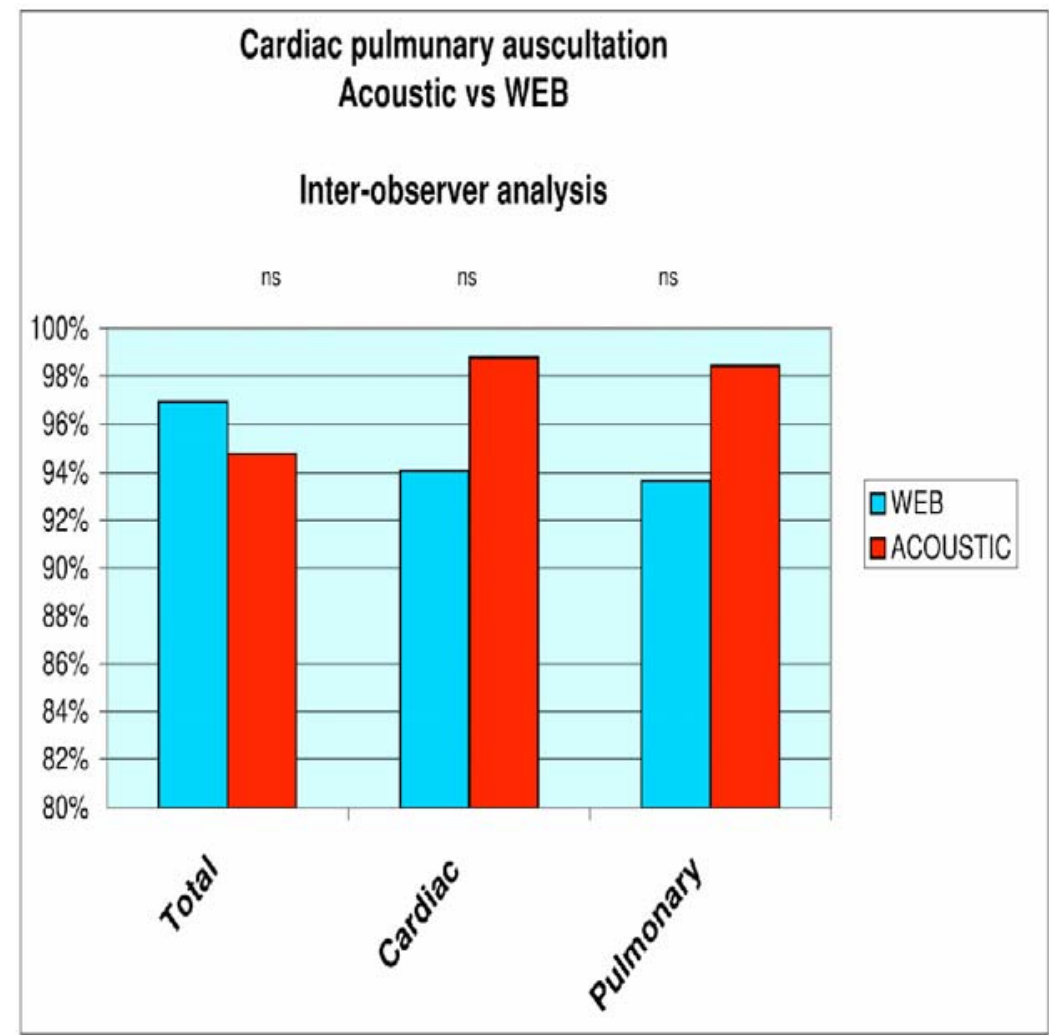

Fig. (5). Histogram of the data taken with the inter-observer analysis.

\section{CONCLUSIONS}

We have proposed a medical electronic-computerized platform for diagnostic use, which allows the doctor to carry out a complete cardio-respiratory control on remote patients in real time.

Moreover the system has been designed to be employed also to real-time rescue in case of emergency without the necessity for data to be constantly monitored by a medical centre, leaving patients free to move. For this purpose the system has been equipped with highly developed firmware which enables automated functioning and complex decisionmaking. In fact, when an emergency sign is detected through the real time diagnosing system, the system sends a warning message to people able to arrange for his/her rescue, providing also the patient coordinates. This occurs automatically without any involvement of the user.

Finally the system, in its version for diagnostic use, has been verified by the heart specialists of the Institute of Cardiology in the General Hospital (Polyclinic) of the University of Bari, Italy.

\section{ACKNOWLEDGEMENTS}

The authors would like to thank Dr. Eng. A. Convertino for his assistance to realize the device prototype and Dr. V.
Marangelli and Prof. S. Favale for their help to verify the system.

\section{REFERENCES}

[1] H. She, Z. Lu, D. Zhou, A. Jantsch, and L. Zheng, "A Networkbased System Architecture for Remote Medical Applications," In Proc. 23 $3^{\text {rd }}$ Asia Pacific Advanced Network Meeting (APAN 2007), Manila, Philippines, 2007.

[2] J. Prolux, R. Clifford, S. Sorensen, D. Lee, and J. Archibald, "Development and evaluation of a Bluetooth EKG monitoring sensor," In Proc. 19 ${ }^{\text {th }}$ International Symposium on Computer Based Medical Systems (CBMS 2006), Salt Lake City, Utah, 2006.

[3] M. Woolard, K. Pitt, A.J. Hayward, and N.C. Taylor, "Limited benefits of Ambulance Telemetry in Delivering Early Thrombolysis: a Randomized Controlled Trial," Emerg. Med. J., vol. 22, pp. 209-215, 2005.

[4] E. Kyriacou, S. Voskarides, C.S. Pattichis, R. Istepanian, M.S. Pattichis, and C.N. Schizas, "Wireless Telemedicine Systems : a brief overview," In Proc. Healthcom 2002, Nancy, France, 2002.

[5] E. Jovanov, A. O’Donnel Lords, D. Raskovic, P.G- Cox, R. Adhami, and F. Andrasik, "Stress monitoring using a distributed wireless intelligent sensor system," IEEE Eng. Med. Biol. Mag., vol. 1, pp. 49-55, 2003.

[6] S. Pavlopoulos, E. Kyriacou, A. Berler, S. Dembeyiotis, and D. Koutsouris "A novel emergency telemedicine system based on wireless communication technology - AMBULANCE," IEEE Trans. Inform. Technol. Biomed., vol. 2, no. 4, pp. 101-107, 1998.

Received: May 25, 2010

Revised: July 26, 2010

Accepted: July 29, 2010

(C) Marani et al.; Licensee Bentham Open.

This is an open access article licensed under the terms of the Creative Commons Attribution Non-Commercial License (http://creativecommons.org/licenses/by-nc/3.0/) which permits unrestricted, non-commercial use, distribution and reproduction in any medium, provided the work is properly cited. 